\title{
Study on Error Analysis Method of Mine Nuclear Belt
}

\author{
Xiu-cai Guo ${ }^{1}$, and Yang $\mathrm{Li}^{1}$
}

${ }^{1}$ College of Electrical and Control Engineering of XUST, Xi'an 710054, China.

\author{
Keywords: Mining Nuclear Belt Scale, The Error of Measurement, Load Factor, Sustainable \\ Exploitation
}

\begin{abstract}
In this paper, the working principle, basic composition and key technologies of mining nuclear belt scale are briefly introduced. The error of measurement is analyzed emphatically, moreover, propose measures to solve or reduce the error. Through the establishment of mining nuclear belt scale error expression model, improve its load factor method to enhance the accuracy of nuclear belt scale, coal mine resources sustainable exploitation.
\end{abstract}

\section{Introduction}

Nuclear belt scale is the mainstream non-contact weighing instrument for dynamic weighing of belt conveyer material. In the early sixties and seventies of the last century, electronic belt scale began to be widely developed, the early 80 s, nuclear belt scale in China began to use[1], but in the actual operating environment, due to underground environmental factors affect the belt scale measurement error, such as the decay of radioactive sources, the shape of the coal accumulation, the ionization chamber surface ash, etc. Some errors can be avoided, so for some avoidance error theoretical analysis and experimental research, and through the establishment of model to improve the accuracy of belt scales to use for reference[2].

\section{Error Analysis of Nuclear Belt Scale}

In the early nuclear belt scale test and calibration are the ideal experimental simulation device, due to differences in coal moisture content and the accumulation of irregular shape, the measurement accuracy will have a greater error, about $\pm 1 \%$. In the actual coal mine application site, the nuclear belt scale work for a long time and the working environment is harsh, making the measurement error is difficult to reduce, in general, the error caused by the following aspects[3]:

(1)The error caused by radioactivity. In a radioactive measurement, the number of atoms decaying in the same radioactive source per unit is different, but the measured value concentrates within a certain range. That is, in the same measurement of material that will fluctuate, the formation of statistical error. Cesium-137 half-life of 30.1671a, the decay of radioactive sources can cause errors.

(2)The intensity of radiation to the ionization chamber is through the average strength of the material, due to the irregular shape of the material, the irregular accumulation of materials in the nuclear belt scale in the work of the actual mathematical model changes, causing uncertain errors.

(3) Due to the limitation of the intensity of radioactive sources, the unit load of the nuclear belt scale also needs to be within a certain range. If the unit load is larger, the radiation intensity decay will be greater, and the signal received by the ionization chamber will be weaker. The smaller the output electrical signal and the accuracy will be reduced. If the unit load is very small, the signal will change small and affect the accuracy, not easy to detect.

(4) Ionization chamber surface ash or skin thickness changes will affect the gamma ray intensity, the measurement results will also have an impact.

In order to improve the accuracy of the belt scale, precise control of coal mining and reduce the error, we analyze avoidable errors, and propose solutions for reference. 


\section{Structure and Working Principle of Nuclear Belt Scale}

Mine nuclear belt scale with a non-contact weighing method to overcome electronic belt scale produced a series of problem, such as belt tension, jitter, deviation and so on[4]. According to the coal mine underground working environment and the actual structure of nuclear belt scale configuration, nuclear scale block diagram shown in Figure 1.

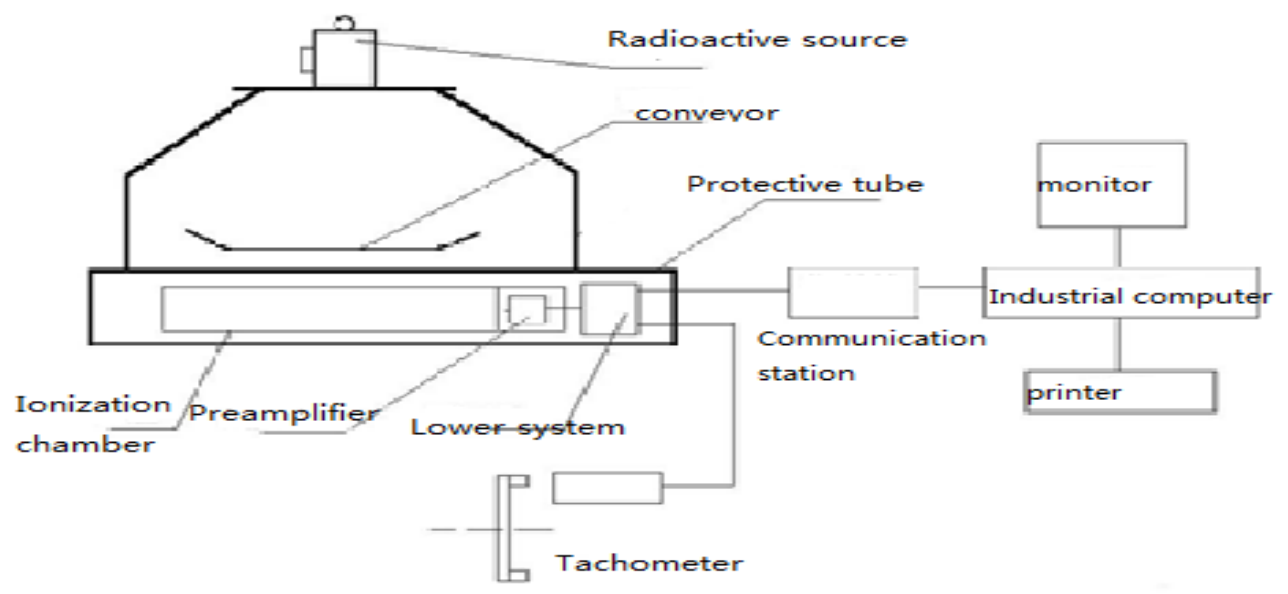

Figure 1.Block diagram of nuclear scale

Nuclear scale is based on the $\gamma$-ray in the penetration properties of materials developed when the absorption: Most of the nuclear scale using Cs137 $\gamma$-ray as a radioactive source, its activity is $40 \times 37 \mathrm{MBq}-100 \times 37 \mathrm{MBq}(40-100 \mathrm{mcc})$, ray energy of $0.66 \mathrm{Mev}$, half-life of 30 years. In operation, the radioactive source emits a fan-shaped $\gamma$-ray stably through a fan-shaped straight hole beneath the lead shield. The $\gamma$-ray passes through the material and is irradiated on the $\gamma$-ray detector (gas ionization chamber) under the material. Precision, long life, simple structure, anti-mechanical damage and so on. The role of the ionization chamber is to irradiate it to different intensity of $\gamma$-ray through the negative pressure into the corresponding weak current, the current size in the $10^{-12} \mathrm{~A}$, the current size reflects the flow of materials, The preamplifier is composed of high gain, high input impedance, low noise, high stability and high precision components. The weak current is amplified and converted into pulse signal to the lower computer system to supply power for the ionization chamber. The lower computer system acquires the data information. The difference between nuclear belt scale and electronic belt scale is the measurement method of material quality. The electronic belt scale uses a variety of pressure sensors to directly measure the unit load within the effective weighing length of the belt scale. Nuclear belt scale measure the unit load through the $\gamma$-ray attenuation of the intensity of the material through the material and the measured material composition, thickness and other functions. Load valueF=K $\times \operatorname{In}\left(U_{0} / U_{1}\right)$ [5]. The load is proportional to the natural logarithm of the ratio of the output voltage signal of the system to the output voltage signal of the system at the time of no-load of the belt, from the above load formula, The load factor of nuclear scale affects the measurement accuracy of nuclear belt scale, but the load factor can't be calculated by the theoretical value of the exact, it is based on the scene of the different physical calibration there are differences, so on-site calibration to obtain the load factor is the only practical method, the nuclear scale is relative measurement [6].

The intensity of the gamma-ray penetrating the material is as follows:

$$
\mathrm{I}_{1}=\mathrm{I}_{0} e^{\mu \mathrm{F} / \mathrm{s}}
$$

Where: $\mu$ is the absorption coefficient of the mass of the $\gamma$-ray material $\left(\mathrm{cm}^{2} / \mathrm{g}\right), \mathrm{F}$ is the belt load, $I_{0}$ is the $\gamma$-ray intensity of the detector at no load, $S$ is the belt width, $I_{1}$ is the gamma ray intensity of the detector at the time of the load.

The $\gamma$-ray detector output voltage signal is proportional to the $\gamma$-ray intensity, it is: 


$$
\mathrm{U}_{1}=\mathrm{U}_{0} \mathrm{e}^{\mu \mathrm{F} / \mathrm{s}}
$$

Where: $U_{0}$ is no-load output voltage signal, $U_{1}$ is the load output voltage signal. $K_{1}$ is the material of the $\gamma$-ray absorption coefficient; $\mathrm{K}_{1}=\mathrm{S} / \mu$

$$
\mathrm{F}=\mathrm{K}_{1} \ln \left(\mathrm{U}_{0} / \mathrm{U}_{1}\right)
$$

Belt conveyor for constant speed operation, that is, a certain speed $\mathrm{V}$, the material flow:

$$
\mathrm{P}=\mathrm{FV}
$$

Where: $\mathrm{P}$ is the material flow rate $\mathrm{t} / \mathrm{h}$; $\mathrm{V}$ is the material conveying speed $\mathrm{m} / \mathrm{s}$.

From this we can calculate the total amount of material W:

$$
\mathrm{W}=\int \mathrm{Pdt}=\sum_{\mathrm{R}=1}^{\mathrm{n}} \mathrm{P}_{\mathrm{R}} \Delta_{\mathrm{t}}=\sum_{\mathrm{R}=1}^{\mathrm{n}} \mathrm{F}_{\mathrm{R}} \mathrm{V}_{\mathrm{R}} \Delta_{\mathrm{t}}
$$

Where: $\mathrm{W}$ is the material accumulation; $\mathrm{P}_{\mathrm{R}}$ is the instantaneous flow of materials, $\Delta_{\mathrm{t}}$ is the sampling period.

\section{Nuclear Belt Scale Error Analysis Model}

From (5) where we can get the error from the following aspects: The error caused by the material load $\mathrm{F}$ and the belt speed, Let $\mathrm{H}_{\mathrm{W}}=\mathrm{dW} / \mathrm{W}$ be the relative error of the material accumulation; $\mathrm{H}_{\mathrm{F}}=\mathrm{dF} / \mathrm{F}$ indicates the relative error of the material load; $\mathrm{H}_{V}=\mathrm{dV} / \mathrm{V}$ indicates the relative error of the measured belt speed; Then according to the above derived (3) and (5):

$$
\begin{aligned}
& \mathrm{H}_{\mathrm{W}}=\mathrm{H}_{\mathrm{F}}+\mathrm{H}_{\mathrm{V}} \\
& \mathrm{H}_{\mathrm{F}}=\frac{\mathrm{dS}}{\mathrm{S}}+\frac{\mathrm{dUp}}{\mathrm{Up}}+\frac{\mathrm{dU} 1(\mathrm{U} 0 / \mathrm{U} 1)}{\ln (\mathrm{U} 1 / \mathrm{U} 0)}
\end{aligned}
$$

From the above expression can be obtained, the nuclear belt scale of the main error from the material mass absorption coefficient, conveyor belt conveyor belt width, no-load output voltage signal, the load output voltage signal error composition. Because the relative error of the belt speed can be avoided with high-precision velocity measurement tools, we mainly discuss the relative error of the material load.

\section{Improve the Error Method}

$Z=\ln \left(U_{1} / U_{0}\right), H_{U}=d U 1 / U 1, Z$ is the load factor of nuclear belt scale that is the size of nuclear belt scale load, $\mathrm{H}_{\mathrm{F}}=\mathrm{H}_{\mathrm{U}} / \mathrm{Z}$. From this formula can be drawn $\mathrm{H}_{\mathrm{F}}$ depends on no-load and load when the output voltage signal [7]. The impact of the output voltage signal is the ionization chamber and preamplifier, so in order to improve the accuracy of nuclear belt scale must develop gas ionization chamber and improve the performance of preamplifier [8]. Select the gas ionization chamber. Its basic working principle is that the ionization of the gas through the material when the ionization of gas molecules, the number of ion pairs produced by the energy loss of the particles. Two electrodes are arranged in the gas ionization chamber, and a certain potential difference is maintained. Positive ions and negative ions are separated by the electric field and drift in the electric field direction, respectively. The charge is collected by the two electrodes and generates a certain electrical signal. Pre-amplifier using I-F transform technology is the current signal into a pulse signal, and the pulse frequency and input current is proportional to the measurement of ionization chamber current.

From the $\mathrm{H}_{\mathrm{U}}$ expression can be seen $\mathrm{U} 1$ larger $\mathrm{H}_{\mathrm{F}}$ smaller, but increase the output voltage U1 
load will make the nuclear belt scale load factor $\mathrm{Z}$ increases, in turn affect the $\mathrm{H}_{\mathrm{F}}$, so that it increases. Therefore, the nuclear belt scale weighing system must have a best coincidence coefficient $Z_{0}$, when $Z=Z_{0}$, the nuclear belt scale from the load will bring the smallest error. Let $\mathrm{W}_{\mathrm{F}}=\frac{\mathrm{H}_{\mathrm{U}}}{\mathrm{Z}}, \mathrm{X}=\frac{\mathrm{U} 1}{\mathrm{U} 0}$, can be simplified by $\mathrm{W}_{\mathrm{F}}=\mathrm{U}_{0} \ln (\ln \mathrm{X})$, MATLAB simulation of the above functions are the following curve, as shown in Figure 4.

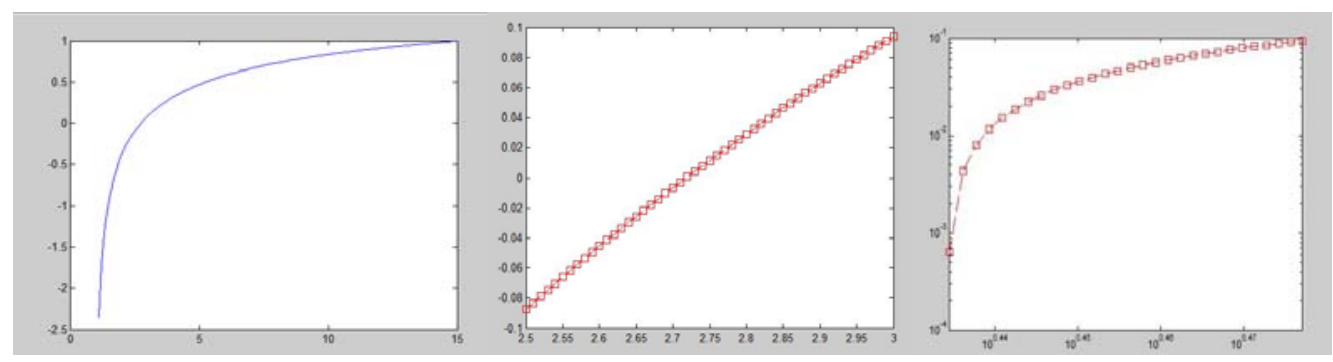

$\mathrm{W}_{\mathrm{F}}$ and $\mathrm{Z}_{0}$ near the simulation curve

Figure 4 MATLAB simulation graphics

It can be seen from the curve there is a minimum $\mathrm{W}_{\mathrm{F}}$, that there is the best load factor $\mathrm{Z}_{0}$ makes the error minimum, In fact from the above expression can be obtained, When $\mathrm{X}=\mathrm{e}$, that is, $\mathrm{U}_{1}=\mathrm{U}_{0} \mathrm{e}$, $\mathrm{W}_{\mathrm{F}}=\mathrm{W}_{\mathrm{Fmin}}=0$. As can be seen from Fig: In order to improve the accuracy of nuclear belt scale, you must choose the appropriate load and no-load voltage ratio, $\mathrm{Z}$ is too large or too small will lead to a sharp increase in error. Only when $\mathrm{Z}$ is maintained near $\mathrm{Z}_{0}$, can guarantee the error will not affect the precision too much.

\section{Conclusion}

In this paper, the principle of the nuclear belt scale and the causes of the error are introduced and the mathematical modeling and simulation of intuitive understanding of the impact of nuclear belt scale accuracy of certain parameters, The setting of the load parameter of the nuclear belt scale, when it is maintained in the vicinity of $\mathrm{Z}_{0}$ will make the belt scale accuracy has improved significantly, in the context of the sustainable development of the coal industry is of great significance.

\section{References}

[1]Fang Yuanbo. Electronic belt scale [M]. Beijing. Metallurgical Industry Press, 2007.

[2]Zhang Shiwei.CCG nuclear belt scale principle and application [J].Industrial measurement, 2002.

[3] Gu Yuan country.Belt conveyor failure analysis and treatment [J].Paper and paper, 2002 (4).

[4] Fang Yuanbo. Comparison of nuclear belt scale and electronic belt scale [J].World Meters and Automation, 2002.

[5] Shi Changyan. Electronic weighing technology status and development trend [J].

[6] Tsinghua University Institute of nuclear energy technology: "QH-200B nuclear belt scale manual."

[7] GanShihong. Error Analysis and Improvement of Microcomputer Nucleon Scales [J].Nuclear Electronics \& Detection Technology, 2005 (3).

[8] Chen Mingzhen. Analysis and control of nuclear scale system error [J]. Industrial measurement 2001, (1): 22-24. 